\title{
On the definition, utility, and practical implementation of X-ray omnidirectional differential phase contrast and dark-field imaging
}

\author{
Matias Kagias ${ }^{a, b, 1}$ and Marco Stampanoni ${ }^{a, c}$
}

The paper by Wang and Sawhney (1) describes the use of a speckle pattern in order to retrieve omnidirectional differential phase and dark-field images. Although the approach is methodologically correct and the claim that a simple modulator can have distinct benefits comparted to X-ray optics is true, there are a few passages in both the significance statement and the main text that we find to be inaccurate.

1) The authors (1) state that "the proposed method enables us to detect the subtle phase changes in all directions of the imaging plane, which complements conventional $\mathrm{X}$-ray imaging methods with information that they cannot provide." We find this statement to be erroneous for differential phase contrast imaging. The explanation for this can be found in the very definition of the directional derivative, which is the mathematical equivalent of the directional differential phase contrast images. From a mathematical perspective, there is no need for retrieving omnidirectional differential phase images, since it is well known that directional derivates can be obtained by having access to two orthogonal components (2). Therefore, if an imaging method can retrieve differential phase contrast images in $x$ and $y$ directions, annotated by $\partial \Phi(x, y) / \partial x$ and $\partial \Phi(x, y) / \partial y$, respectively, the directional derivative under any angle $\theta$ on the imaging plane is given by $\cos (\theta) \partial \Phi(x, y) / \partial x+\sin (\theta)$ $\partial \Phi(x, y) / \partial y$. It should be pointed out that a variety of already published modulator-based imaging methods (i.e., grating interferometry, coded apertures, and speckle-based imaging) can provide direct access to the two orthogonal components with or without scanning optical elements, and hence omnidirectional differential phase images can be produced by existing methodology. It is not clear whether the reported approach exhibits any benefit by retrieving the directional differential phase contrast images with the proposed algorithm. At least a discussion or quantitative comparison would be necessary in order to render the claim believable. For instance, does the proposed method produce higher signal-to-noise ratio? Is there something truly gained from a physical point of view?

2) Furthermore, it is stated that "the presented technique could potentially open up numerous practical imaging applications in both biomedical research and materials science." This seems to imply that the already published methodology for omnidirectional scattering imaging is limited and lacking practical implementations. However, recent developments have allowed practical implementations of omnidirectional scattering imaging with applications in real-life scenarios. In particular, the publication by Kagias et al. (3) shows how a general design of X-ray optics enables a robust omnidirectional scattering imaging approach. The method is compatible with a wide variety of X-ray sources (synchrotron, microfocal and macrofocal $X$-ray tubes) and requires a single optical element, and-in addition to omnidirectional dark-field images_-it also provides differential phase contrast images in the $x$ and $y$ directions (which, as stated earlier, are equivalent to omnidirectional differential phase contrast imaging). Furthermore, the work in Kagias et al. (3) shows an application of the method on industrially relevant samples (glass and carbon fiber-reinforced polymers) in addition to real-time imaging.

1 H. Wang, K. Sawhney, Hard X-ray omnidirectional differential phase and dark-field imaging. Proc. Natl. Acad. Sci. U.S.A. 118, e2022319118 (2021).

2 W. Kaplan, "The directional derivative" in Advanced Calculus (Addison-Wesley, Reading, MA, ed. 4, 1991), pp. 135-138.

3 M. Kagias et al., Diffractive small angle X-ray scattering imaging for anisotropic structures. Nat. Commun. 10, 5130 (2019).

${ }^{a}$ Swiss Light Source, Paul Scherrer Institute, Villigen 5232, Switzerland; 'b Division of Engineering and Applied Science, California Institute of

Technology, Pasadena, CA 91125; and 'Institute for Biomedical Engineering, University of Zurich and ETH Zurich, Zurich 8092, Switzerland

Author contributions: M.K. and M.S. wrote the paper.

The authors declare no competing interest.

Published under the PNAS license.

${ }^{1}$ To whom correspondence may be addressed. Email: mkagias@caltech.edu.

Published November 15, 2021. 